\title{
Charmless two- and four-body b-hadron decays at $\mathrm{LHCb}$
}

\section{María Vieites Díaz ${ }^{* \dagger}$}

Universidade de Santiago de Compostela

E-mail: maria.vieites.diazecern.ch

In this document, two of the latest results obtained by the LHCb Collaboration on the area of charmless $b$ decays are summarised. The first observation of the very rare $\mathrm{B}^{0} \rightarrow \mathrm{K}^{+} \mathrm{K}^{-}$decay mode is presented, together with the most precise measurements of $\mathscr{B}\left(\mathrm{B}^{0} \rightarrow \mathrm{K}^{+} \mathrm{K}^{-}\right)$and $\mathscr{B}\left(\mathrm{B}_{s}^{0} \rightarrow\right.$ $\pi^{+} \pi^{-}$). The second result is related to $C P$ violation in baryonic matter. A search for $P$ and $C P$ violation was performed by studying triple-product asymmetries in the $\Lambda_{b}^{0} \rightarrow p \pi^{-} \pi^{+} \pi^{-}$and $\Lambda_{b}^{0} \rightarrow p \pi^{-} \mathrm{K}^{+} \mathrm{K}^{-}$decay channels. This leads to the first evidence of matter-antimatter asymmetry in the baryonic sector.

38th International Conference on High Energy Physics 3-10 August 2016

Chicago, USA

*Speaker.

On behalf of the LHCb Collaboration. 


\section{Introduction}

Within the Standard Model (SM) framework, charmless $b$ decays proceeding via tree diagrams are suppressed by the smallness of the $\left|V_{u b}\right|$ term in the CKM matrix. Their contribution to the final decay rate is, therefore, of a similar order of magnitude of the otherwise sub-leading penguin process. This situation enhances the interference effects between the two processes and allows the measurement of their relative weak phase difference. The relative importance of the penguin diagrams in charmless processes could allow the detection of new particles entering in the loops which would cause deviations from the branching fractions and $C P$ asymmetries predicted by the SM.

Both analyses presented here have used the full data sample from the Run1 of $\mathrm{LHCb}$, corresponding to $1 f b^{-1}$ at $\sqrt{s}=7 \mathrm{TeV}$ and $2 f b^{-1}$ at $\sqrt{s}=8 \mathrm{TeV}$ of $p p$ collisions. The LHCb detector consists of a single-arm forward spectrometer specifically designed for flavour physics. The characteristics of this design include a very good vertex and tracking resolution, a fully instrumented forward coverage that maximizes the acceptance of the $b \bar{b}$ quark pairs produced at the LHC and a very efficient particle identification (PID) system separating protons, pions and kaons in the full acceptance. Together with a high performing trigger, these vertexing and PID efficiencies are crucial in order to study flavour physics in the very complicated hadronic environment of the LHC. Further details about the detector can be found in [1].

\section{The $\mathrm{B}^{0} \rightarrow \mathrm{K}^{+} \mathrm{K}^{-}$and $\mathrm{B}_{s}^{0} \rightarrow \pi^{+} \pi^{-}$decays}

The $\mathrm{B}^{0} \rightarrow \mathrm{K}^{+} \mathrm{K}^{-}$decay is the rarest fully hadronic $b$ meson decay ever observed [2]. The measurement of the branching fraction of its U-spin partner, the $\mathrm{B}_{s}^{0} \rightarrow \pi^{+} \pi^{-}$, has been updated by the LHCb collaboration, achieving an unprecedented precision. Figure 1 shows the leading diagrams contributing to the $\mathrm{B}^{0} \rightarrow \mathrm{K}^{+} \mathrm{K}^{-}$and the $\mathrm{B}_{s}^{0} \rightarrow \pi^{+} \pi^{-}$decay amplitudes. These picture a penguin annihilation (PA) and a W-exchange process, which are both very suppressed in the SM and of great interest to understand the dynamics governing the decays of heavy-flavoured hadrons.

The search for the $\mathrm{B}^{0} \rightarrow \mathrm{K}^{+} \mathrm{K}^{-}$mode was performed following a blind analysis strategy, in which the regions $5.22<m_{K K}<5.34 \mathrm{GeV} / \mathrm{c}^{2}$ and $5.33<m_{\pi \pi}<5.45 \mathrm{GeV} / \mathrm{c}^{2}$ are disregarded during selection optimization. In order to be selected, candidates must satisfy hardware and software trigger requirements. The former implies having large transverse energy clusters in the hadronic calorimeter, and the latter a well reconstructed and displaced secondary vertex (SV) and, at least, one charged track with a large impact parameter (IP) with respect to all the $p p$ interaction points above given thresholds. A multivariate classifier (Boosted Decision Tree, BDT [3]) is used offline
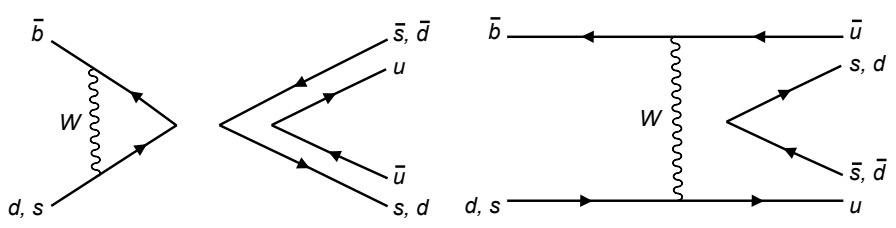

Figure 1: Dominant diagrams contributing to the $\mathrm{B}^{0} \rightarrow \mathrm{K}^{+} \mathrm{K}^{-}$and $\mathrm{B}_{s}^{0} \rightarrow \pi^{+} \pi^{-}$decay amplitudes. The processes pictured here correspond to penguin annihilation (left) and W-exchange (right). 
in order to compute for each $B$ candidate its likelihood for being signal or background. The BDT classifier obtains its discriminating power from the same variables that were used in the trigger, together with the IP $\chi^{2}$ of the B meson, the goodness of the SV fit and information on the separation of the SV from the PV. The PID hypothesis mainly relies on the information collected by the Ring Imaging Cherenkov (RICH) detectors and it is used to classify the events in mutually exclusive subsamples, according to the hadrons in the final state $(\mathrm{K} \pi, \mathrm{pK}, \mathrm{p} \pi, \pi \pi$ and $\mathrm{KK})$. Event migration among the different samples (cross-feed backgrounds) has to be very well controlled in order to be able to measure each yield with a good precision. The PID calibration is performed following a data-driven method, which uses samples of pions and kaons from the easily identified $D^{*+}, \Lambda$ and $\Lambda_{c}^{+}$decays. At the final step of the selection process, BDT and PID requirements are optimized simultaneously aiming at the best sensitivity on the signal yield. This optimization is computed twice, once for each signal $\left(\mathrm{B}^{0} \rightarrow \mathrm{K}^{+} \mathrm{K}^{-}\right.$or $\left.\mathrm{B}_{s}^{0} \rightarrow \pi^{+} \pi^{-}\right)$mode.

The final yields are obtained from a simultaneous 2-body invariant mass fit to all the selected subsamples. Figure 2 shows the fit projections in the $K K$ and the $\pi \pi$ invariant mass spectra, where the distributions corresponding to the two signal models are drawn with a filled curve. In order to compute the systematic uncertainties, the results of this fit are used to generate pseudo-experiments that are then fitted back with alternative mass shapes. The difference in the signal yield results between the fit with the nominal model and the alternative ones is used as systematic uncertainty. The final branching ratios determination are performed using the HFAG average of $\mathscr{B}\left(\mathrm{B}_{s}^{0} \rightarrow \mathrm{K}^{+} \pi^{-}\right)$ [4] as normalisation and the best known value of $\left(f_{s} / f_{d}\right)$ [5], finding

$$
\begin{aligned}
& \mathscr{B}\left(\mathrm{B}^{0} \rightarrow \mathrm{K}^{+} \mathrm{K}^{-}\right)=(7.80 \pm 1.27 \text { (stat) } \pm 0.81 \text { (syst) } \pm 0.21(\text { norm })) \times 10^{-8} \\
& \mathscr{B}\left(\mathrm{B}_{s}^{0} \rightarrow \pi^{+} \pi^{-}\right)=\left(6.91 \pm 0.54 \text { (stat) } \pm 0.63 \text { (syst) } \pm 0.19(\text { norm }) \pm 0.40\left(f_{s} / f_{d}\right)\right) \times 10^{-7} .
\end{aligned}
$$

These results correspond to the first observation of the $\mathrm{B}^{0} \rightarrow \mathrm{K}^{+} \mathrm{K}^{-}$mode, in which a significance of $5.8 \sigma$ was achieved, and the most precise measurement up to date of $\mathscr{B}\left(\mathrm{B}_{s}^{0} \rightarrow \pi^{+} \pi^{-}\right)$.

\section{The $\Lambda_{b}^{0} \rightarrow p \pi^{-} \pi^{+} \pi^{-}$and $\Lambda_{b}^{0} \rightarrow p \pi^{-} \mathrm{K}^{+} \mathrm{K}^{-}$decays}

According to the SM predictions, a sizeable $C P$ violation effect should occur in charmless decays of baryonic matter. Asymmetries as large as $\mathscr{A}_{C P} \sim 20 \%$ have been predicted for $\Lambda_{b}^{0}$ decays
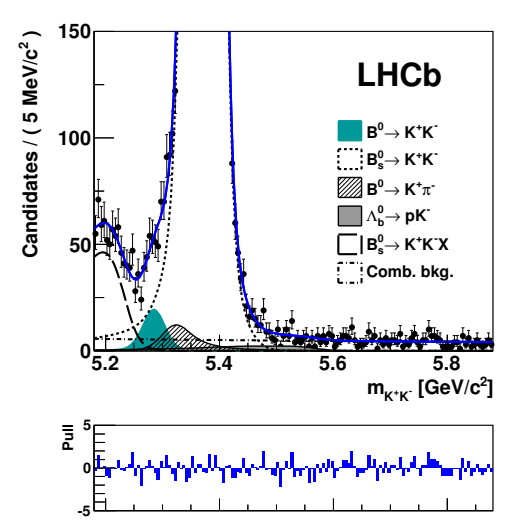
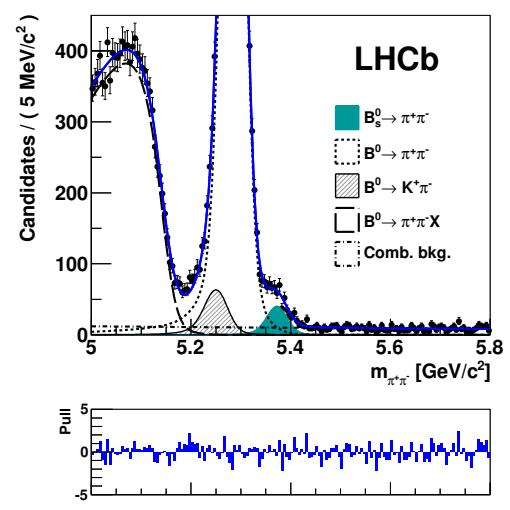

Figure 2: Fit projections in $m_{K K}$ (left) and $m_{\pi \pi}$ (right) containing the signal modes. The solid blue line represents the projection of the total model and the two filled curves, the signal decays. 

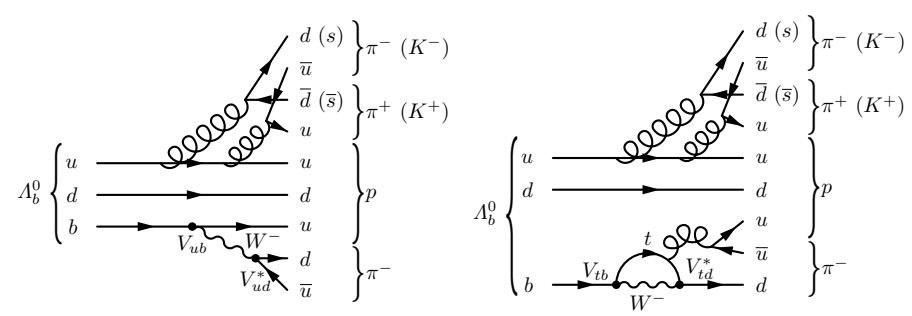

Figure 3: Tree (left) and penguin (right) Feynman diagrams governing the $\Lambda_{b}^{0} \rightarrow p \pi^{-} h^{+} h^{-}$ $(h=\pi, \mathrm{K})$ decays. The similar magnitude of these processes (tree $\propto\left|V_{u b}\right| \sim \lambda^{3}$ and penguin $\propto \sum_{x=u, c, t} V_{b x} V_{x d} \sim \lambda^{3}$ ) enhances the interference between them, and thus, the $C P$ violating effect.

[6] and their measurement can be used to test the SM consistency and to place constraints on possible extensions of this model. Evidence of such effects has been seen after performing a search for $P$ and $C P$ violation in $\Lambda_{b}^{0} \rightarrow p \pi^{-} h^{+} h^{-}(h=\pi, \mathrm{K})$ decays using triple product asymmetries [7]. The leading Feynman diagrams contributing to these decays are shown in Figure 3.

The observables used for this analysis are constructed as follows:

1. The triple products in the $\Lambda_{b}^{0}$ rest frame are: $C_{\hat{T}}=\vec{p}_{p} \cdot\left(\vec{p}_{h^{-}} \times \vec{p}_{h^{+}}\right) \propto \sin \Phi$ and $\bar{C}_{\hat{T}}=\vec{p}_{\bar{p}}$. $\left(\vec{p}_{h^{+}} \times \vec{p}_{h^{-}}\right) \propto \sin \bar{\Phi}$. For the $\Lambda_{b}^{0} \rightarrow p \pi^{-} \pi^{+} \pi^{-}$decay, the pion with the highest momentum in the $\Lambda_{b}^{0}$ rest frame $\left(\pi_{\text {fast }}\right)$ is chosen for the computation (see Figure 4 ).

2. From these, two $\hat{T}-$ odd asymmetries can be built as:

$$
A_{\hat{T}}=\frac{N_{\Lambda_{b}^{0}}\left(C_{\hat{T}}>0\right)-N_{\Lambda_{b}^{0}}\left(C_{\hat{T}}<0\right)}{N_{\Lambda_{b}^{0}}\left(C_{\hat{T}}>0\right)+N_{\Lambda_{b}^{0}}\left(C_{\hat{T}}<0\right)} \quad \text { and } \quad \bar{A}_{\hat{T}}=\frac{N_{\bar{\Lambda}_{b}^{0}}\left(-\bar{C}_{\hat{T}}>0\right)-N_{\bar{\Lambda}_{b}^{0}}\left(-\bar{C}_{\hat{T}}<0\right)}{N_{\bar{\Lambda}_{b}^{0}}\left(-\bar{C}_{\hat{T}}>0\right)+N_{\bar{\Lambda}_{b}^{0}}\left(-\bar{C}_{\hat{T}}<0\right)},
$$

where $\hat{T}$ is the motion reversal operator that reverts both momentum and spin three-vectors and $N_{\Lambda_{b}^{0}}\left(N_{\bar{\Lambda}_{b}^{0}}\right)$ is the number of $\Lambda_{b}^{0}\left(\bar{\Lambda}_{b}^{0}\right)$ signal events. It should be noted that these quantities are, by construction, insensitive to particle/antiparticle production asymmetries and to detector-induced charge asymmetries [8].

3. Finally, the $C P$ and $P$ violating observables can be constructed from these asymmetries as:

$$
a_{C P}^{\hat{T}-\text { odd }}=\frac{1}{2}\left(A_{\hat{T}}-\bar{A}_{\hat{T}}\right) \quad \text { and } \quad a_{P}^{\hat{T}-o d d}=\frac{1}{2}\left(A_{\hat{T}}+\bar{A}_{\hat{T}}\right),
$$

where observing a significant deviation from zero in any of their values would indicate a sign for $C P$ or $P$ violation, respectively.

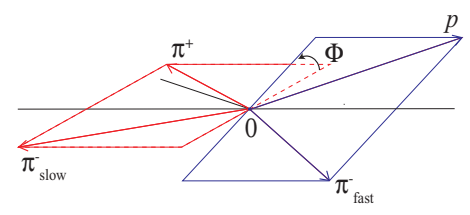

Figure 4: Definition of the helicity angles in the $\Lambda_{b}^{0}$ decays. The pion with the highest (lowest) momentum in this reference frame is referred to as $\pi_{\text {fast }}\left(\pi_{\text {slow }}\right)$. 

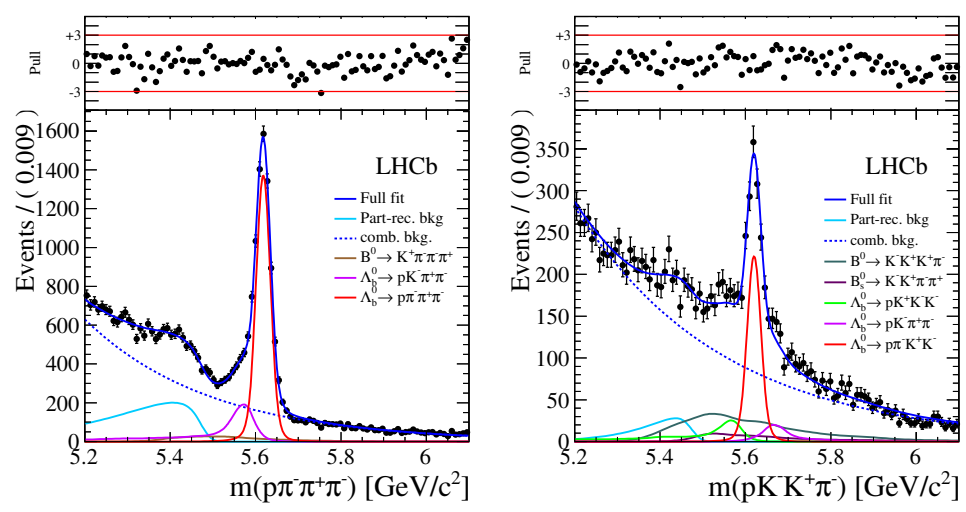

Figure 5: Invariant mass distributions for the $\Lambda_{b}^{0} \rightarrow p \pi^{-} \pi^{+} \pi^{-}$(left) and the $\Lambda_{b}^{0} \rightarrow p \pi^{-} \mathrm{K}^{+} \mathrm{K}^{-}$ (right) signal candidates.

Table 1: $P$ - and $C P$-violating observables obtained from the fit to the whole data sample.

\begin{tabular}{ccc}
\hline Decay & $a_{P}^{\hat{T}-\text { odd }}[\%]$ & $a_{C P}^{\hat{T}-\text { odd }}[\%]$ \\
\hline$\Lambda_{b}^{0} \rightarrow p \pi^{-} \pi^{+} \pi^{-}$ & $-3.71 \pm 1.45 \pm 0.32$ & $1.5 \pm 1.45 \pm 0.32$ \\
$\Lambda_{b}^{0} \rightarrow p \pi^{-} K^{+} K^{-}$ & $3.62 \pm 4.54 \pm 0.42$ & $-0.93 \pm 4.54 \pm 0.42$ \\
\hline
\end{tabular}

The analysed data sample is selected following a similar strategy to that defined in the previous section. $\Lambda_{b}^{0} \rightarrow p \pi^{-} h^{+} h^{-}$signal candidates are reconstructed combining proton, pion and kaon tracks above a certain momenta threshold. The charge of the proton in the final state is used to identify the flavour of the decaying $\Lambda_{b}^{0}$ and this, together with the sign of $C_{\hat{T}}\left(\bar{C}_{\hat{T}}\right)$, allows to split the data in four different categories. The signal yields are then obtained from a simultaneous fit to the $M\left(p \pi^{-} h^{+} h^{-}\right)$spectra, which result is shown in Figure 5. A total of $6646 \pm 105$ and $1030 \pm 56$ events are found for the $\Lambda_{b}^{0} \rightarrow p \pi^{-} \pi^{+} \pi^{-}$and the $\Lambda_{b}^{0} \rightarrow p \pi^{-} \mathrm{K}^{+} \mathrm{K}^{-}$modes, respectively, corresponding to the first observation of these decays. The results obtained for the two observables are summarized in Table 1 and are consistent with $P$ and $C P$ symmetry.

The larger size of the $\Lambda_{b}^{0} \rightarrow p \pi^{-} \pi^{+} \pi^{-}$data sample allows for a refined search for $C P$ violation effects. The variation of the strong phases along the phase space and the possibility of observing $C P$ asymmetry in the angular distributions of quasi-two-body decay amplitudes motivated the use of two different binning schemes to perform a localised search. The first binning scheme defines 12 regions dominated by 2 -body resonances $\left(\rho^{0}(770), \Delta^{++}, N^{*}\right)$, whereas the second one consists of 10 uniform bins in the $\Phi$ angle. Figure 6 shows $a_{P}^{\hat{T}-o d d}$ and $a_{C P}^{\hat{T}-o d d}$ in these $10 \Phi$ bins for the $\Lambda_{b}^{0} \rightarrow p \pi^{-} \pi^{+} \pi^{-}$decay, together with the projection of the 4-body invariant mass fit for the bin which shows the largest deviation from zero. The measured asymmetries in this bin are $A_{\hat{T}}=$ $(19.47 \pm 6.96 \pm 0.86) \%, \bar{A}_{\hat{T}}=(20.11 \pm 7.04 \pm 0.85) \%$, and $a_{C P}^{\hat{T}-\text { odd }}=(19.79 \pm 4.95 \pm 0.60) \%$.

\section{Conclusions}

The first observation of the $\mathrm{B}^{0} \rightarrow \mathrm{K}^{+} \mathrm{K}^{-}$decay was achieved with a significance of $5.8 \sigma$. The most precise measurements of $\mathscr{B}\left(\mathrm{B}_{s}^{0} \rightarrow \pi^{+} \pi^{-}\right)$and $\mathscr{B}\left(\mathrm{B}^{0} \rightarrow \mathrm{K}^{+} \mathrm{K}^{-}\right)$were reported, and are expected to provide a very useful reference for a better understanding of the QCD effects involving PA 

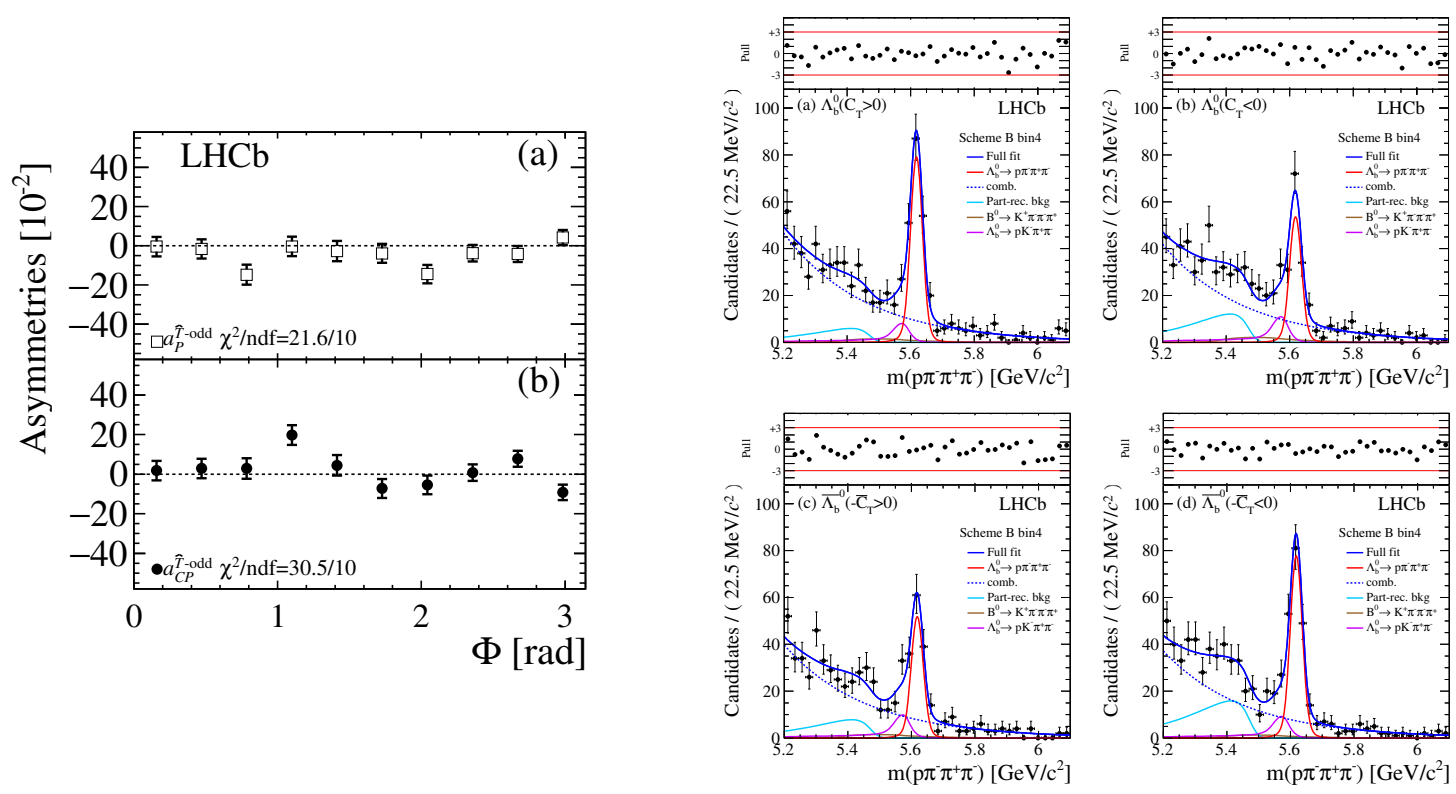

Figure 6: Left: measurements of the $a_{P}^{\hat{T}-o d d}$ and $a_{C P}^{\hat{T}-o d d}$ asymmetries in the $10 \Phi$ bins. Right: fit projections for the four signal categories bin showing the largest deviation $\left(\frac{3}{10} \pi<\Phi<\frac{4}{10} \pi\right)$.

diagrams. $C P$ violation has been searched for using the $\Lambda_{b}^{0} \rightarrow p \pi^{-} \mathrm{K}^{+} \mathrm{K}^{-}$and the $\Lambda_{b}^{0} \rightarrow p \pi^{-} \pi^{+} \pi^{-}$ decays. The results obtained for the $\Lambda_{b}^{0} \rightarrow p \pi^{-} \mathrm{K}^{+} \mathrm{K}^{-}$are compatible with $P$ and $C P$ conservation, while the $\Lambda_{b}^{0} \rightarrow p \pi^{-} \pi^{+} \pi^{-}$channel shows evidence for localised $C P$ violation (3.3 $\sigma$ when combining results from different binning schemes). Both results are in agreement with SM predictions.

\section{References}

[1] Roel Aaij et al. [LHCb collaboration] LHCb Detector Performance. Int. J. Mod. Phys., A30(07):1530022, 2015.

[2] Roel Aaij et al. [LHCb collaboration] Observation of the annihilation decay mode $\mathrm{B}^{0} \rightarrow \mathrm{K}^{+} \mathrm{K}^{-}$

[3] L. Breiman, J. H. Friedman, R. A. Olshen, and C. J. Stone, Classification and regression trees, Wadsworth international group, Belmont, California, USA, 1984.

[4] Heavy Flavor Averaging Group, Y. Amhis et al., Averages of b-hadron, c-hadron, and $\tau$-lepton properties as of summer 2014

[5] R. Aaij et al. [LHCb collaboration] Measurement of the fragmentation fraction ratio fs/fd and its dependence on B meson kinematics

[6] Y. K. Hsiao and C. Q. Geng, Direct CP violation in $\Lambda_{b}^{0}$ decays, Phys. Rev. D91 (2015), no. 11116007

[7] Roel Aaij et al. [LHCb Collaboration] Measurement of matter-antimatter differences in beauty baryon decays

[8] Roel Aaij et al. [LHCb Collaboration] earch for CP violation using T-odd correlations in $D^{0} \rightarrow K^{+} K^{-} \pi^{+} \pi^{-}$decays, JHEP 10 (2014) 005 